\title{
Characterizing Villitis of Unknown Etiology and Inflammation in Stillbirth
}

\author{
Hayley Derricott, ${ }^{*}$ Rebecca L. Jones, ${ }^{*}$ Susan L. Greenwood, ${ }^{*}$ Gauri Batra, ${ }^{\dagger}$ Margaret J. Evans, ${ }^{\ddagger}$ and Alexander E.P. Heazell ${ }^{*}$
}

From the Maternal and Fetal Health Research Centre,* Institute of Human Development, University of Manchester, Manchester Academic Health Science Centre, Manchester; the Department of Paediatric Histopathology, ${ }^{\dagger}$ Royal Manchester Children's Hospital, Manchester; and the Department of Paediatric Histopathology, ${ }^{\ddagger}$ Edinburgh Royal Infirmary, Edinburgh, United Kingdom

\author{
Accepted for publication \\ December 8, 2015. \\ Address correspondence to \\ Hayley Derricott, Ph.D., \\ Department of Infection \\ Biology, Liverpool Science \\ Park IC2, 146 Brownlow Hill, \\ Liverpool L3 5RF, United \\ Kingdom. E-mail: \\ hayleyderricott@live.co.uk.
}

\begin{abstract}
Villitis of unknown etiology (VUE) is an enigmatic inflammatory condition of the placenta associated with fetal growth restriction and stillbirth. Greater understanding of this condition is essential to understand its contribution to adverse outcomes. Our aim was to identify and quantify the cells in VUE in cases of stillbirth and to characterize immune responses specific to this condition. Immunohistochemistry was performed on placentas from stillborn infants whose cause of death was recorded as VUE to identify $\mathrm{CD} 45^{+}$leukocytes, $\mathrm{CD}_{163}{ }^{+}$macrophages, $\mathrm{CD}^{+}$and $\mathrm{CD}^{+} \mathrm{T}$ cells, neutrophils, and proinflammatory and anti-inflammatory cytokines. Images were quantified with HistoQuest software. CD45 leukocytes comprised $25 \%$ of cells in VUE lesions: macrophages (12\%) and CD4 T cells (11\%) being predominant cell types; CD8 T cells were observed in all lesions. Leukocytes and macrophages were increased throughout the placenta in stillbirths; pan-placental $C D 4^{+}$and $\mathrm{CD}^{+} \mathrm{T}$ cells outside VUE lesions were increased in stillbirth with VUE. There was increased IL-2 and IL-12 and reduced IL-4 immunostaining in VUE lesions. Our results suggest VUE in stillbirth has a similar immune cell profile to live birth. Pan-placental macrophages, CD4 and CD8 T cells indicate a wider inflammatory response unrestricted to VUE lesions. The cytokine profile observed suggests a skew towards inappropriate Th1 immune responses. Full characterisation VUE lesion phenotype confirms its immunological origins and provides foundations to develop novel investigations. (Am J Pathol 2016, 186: 952-961; http:// dx.doi.org/10.1016/j.ajpath.2015.12.010)
\end{abstract}

Stillbirth, the death of a baby after 20 weeks' gestation, places a sustained economic and emotional burden on both maternity services and the wider community. Despite significant effort, the stillbirth rate has decreased at a slower rate than neonatal or infant death. ${ }^{1}$ In high-income countries stillbirth rates are variable. The United States and United Kingdom perform relatively poorly with rates of 1 in 160 and 1 in 212, respectively, whereas in Finland 1 in 400 pregnancies result in a stillborn infant. $^{2-4}$

Placental dysfunction as a cause of stillbirth has increasingly become a focus of research effort with recognition that placental disorders are the cause of death in up to $65 \%$ of stillbirths. ${ }^{5}$ However, the placental lesions associated with stillbirth are extremely varied and poorly defined, and their relation to pathologic processes leading to stillbirth is difficult to assess. ${ }^{6,7}$ Placental lesions associated with stillbirth include those with genetic, environmental, infective, inflammatory, mechanical, metabolic, and vascular origins. ${ }^{6}$

Understanding of inflammatory disorders of the placenta remains relatively limited in comparison with vascular lesions. Two main inflammatory conditions have been linked to placental dysfunction in the absence of infection: villitis of unknown etiology (VUE) and chronic histiocytic intervillositis. $^{8}$ VUE is an inflammatory condition of the placenta reported to occur in up to $15 \%$ of term placentas and more frequently in pregnancies resulting in poor outcome. ${ }^{9,10}$ The origin of VUE is unclear, although it is proposed to be maternal immune rejection of a semiallogeneic placenta. ${ }^{11-13}$ VUE is identified and characterized by the presence of elevated numbers of fetal macrophages (Hofbauer cells) and an infiltrate

Supported by Tommy's-the Baby Charity (H.D.).

Disclosures: None declared. 
Table 1 Antibodies and Positive Control Tissues Used for Identifying Immune Cell Populations and Cytokines in Placental Villous Tissue

\begin{tabular}{|c|c|c|c|c|c|}
\hline Antigen & Antigen retrieval & Supplier & Working concentration $(\mu \mathrm{g} / \mathrm{mL})$ & Ab type & Control tissue \\
\hline CD45 & Sodium citrate & Dako & 0.5 & $\mathrm{MmAb}$ & Tonsil \\
\hline CD163 & Sodium citrate & AbD Serotec & 10 & $\mathrm{MmAb}$ & Tonsil \\
\hline CD4 (clone 4B12) & Tris-EDTA or sodium citrate & Dako & 4.6 & $\mathrm{MmAb}$ & Tonsil \\
\hline CD8 & Tris-EDTA & Dako & 1.6 & $M \mathrm{mAb}$ & Tonsil \\
\hline NE & None & Dako & 1.1 & $M \mathrm{mAb}$ & PTLI \\
\hline $\mathrm{IL}-2$ & Sodium citrate & Novus Biologicals & 4.5 & $\mathrm{R} p A b$ & Tonsil \\
\hline IL-10 & None & Abcam & 5 & $M \mathrm{mAb}$ & Liver (Kupffer cells) \\
\hline IL-12 & Sodium citrate & Sigma-Aldrich & 2.5 & $\mathrm{R} p A b$ & Trophoblast \\
\hline TGF- $\beta$ & Sodium citrate & Novus Biologicals & 10 & $M \mathrm{mAb}$ & Tonsil \\
\hline
\end{tabular}

M mAb, mouse monoclonal antibody; NE, neutrophil elastase; PTLI, preterm labor with infection; R pAb, rabbit polyclonal antibody; TGF, transforming growth factor.

of maternal T lymphocytes in the villous stroma. Areas of the placenta not affected by VUE lesions display apparently normal structural characteristics. ${ }^{9}$ The cell profile of VUE has previously been characterized in both appropriately grown and growth-restricted live born infants. ${ }^{12,14-16}$ Conflicting reports state either $\mathrm{CD}^{+}{ }^{+}$or $\mathrm{CD}^{+}{ }^{+} \mathrm{T}$ cells are the most common lymphocyte type in VUE lesions. ${ }^{12,14,17,18}$ The subtype of $\mathrm{CD}^{+}$cells (Th1/Th2) has not been examined; therefore, the type of immune response in VUE remains to be elucidated.

A thorough characterization of VUE has yet to be completed in stillbirth; it is not understood why it may cause fetal demise. In addition, the cytokine profile in VUE has not been described in either live births or stillbirths. Characterization of the cell types present and their cytokine profile will provide a greater understanding of the pathophysiology of VUE. We hypothesized that the immune cells in VUE lesions in stillbirth would be comparable with those described in live birth and that evidence of widespread inflammatory changes would be detectable in the placentas of stillborn infants with VUE. In this immunomorphologic study we aimed to accurately quantify the type and number of immune cells present in VUE lesions, assess leukocyte infiltration across the placenta, and analyze the expression of a range of proinflammatory and anti-inflammatory cytokines with the use of immunohistochemical approaches, coupled with an unbiased sensitive image analysis method. We compared leukocyte infiltration and cytokine expression across the placenta to ascertain whether this was altered in dysfunctional placentas from stillbirth with and without VUE and fetal growth restriction (FGR) compared with healthy controls.

\section{Materials and Methods}

\section{Study Population}

Placental tissues from pregnancies resulting in stillbirth in which a contributory cause of death was recorded as VUE $(n=12)$ or FGR $(n=12)$ were obtained from the Pediatric Histopathology departments of Manchester Royal Children's Hospital and Edinburgh Royal Infirmary. Samples from stillborn growth-restricted infants had no evidence of VUE lesions recorded on postmortem examination. Consent for tissue use in research was obtained at the time of consent for postmortem; their use in this project was approved by Humber Bridge Research Ethics Committee (Ref. 13/YH/0176). Live born infants $(n=11)$ with an individualized birth weight centile less than the fifth were considered FGR infants; placental tissue was obtained from the Maternal and Fetal Health Research Group Biobank, University of Manchester (North West REC 08/H1010/55). Matched placental samples from women with uncomplicated pregnancies $(n=12)$ were selected from the Maternal and Fetal Health Research Group

Table 2 Demographic Information for the Women Included in the Study

\begin{tabular}{|c|c|c|c|c|c|}
\hline Characteristic & $\begin{array}{l}\text { Stillbirth with } \\
\text { VUE }(n=12)\end{array}$ & $\begin{array}{l}\text { Stillbirth with FGR } \\
(n=12)\end{array}$ & $\begin{array}{l}\text { Live birth with } \\
\text { FGR }(n=11)\end{array}$ & $\begin{array}{l}\text { Normal pregnancy } \\
(n=12)\end{array}$ & $P$ \\
\hline Maternal age (years) & $32(21-36)$ & $23.5(19-42)^{*}$ & $32(16-35)$ & $35(25-42)$ & 0.019 \\
\hline Gravidity & $3(1-7)(n=7)$ & $1(1-4)$ & $1(1-9)$ & $2(1-18)$ & 0.298 \\
\hline Parity & $1(0-3)(n=7)$ & $0(0-2)$ & $0(0-3)$ & $1(0-3)$ & 0.118 \\
\hline Birthweight (g) & NA & $1061(385-2000)^{* * *}$ & $2246(747-3060)^{* *}$ & $3430(2900-3840)$ & $<0.001$ \\
\hline
\end{tabular}

Results are presented as median (range).

${ }^{*} P<0.05,{ }^{* *} P<0.01$, and ${ }^{* * *} P<0.001$ compared with normal pregnancy.

FGR, fetal growth restriction; IBC, individualized birth weight centile; NA, not available; VUE, Villitis of unknown etiology. 


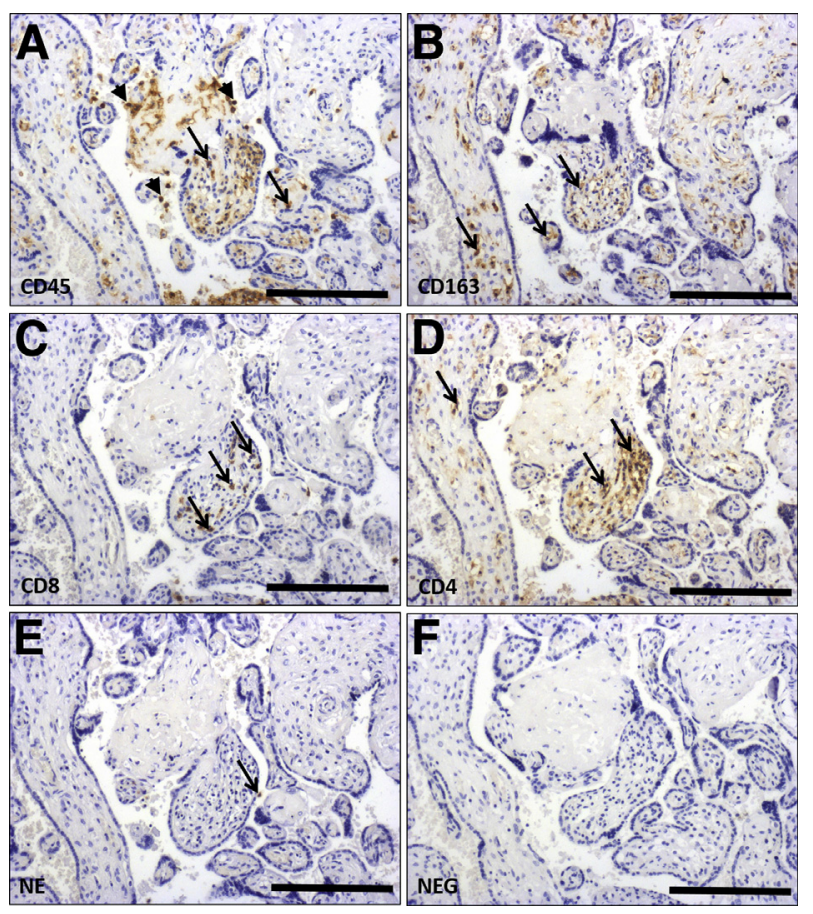

Figure 1 Serial images of a representative VUE lesion immunostained for immune cell markers. CD45 (A), CD163 ${ }^{+}$macrophages (B), CD8 cytotoxic $\mathrm{T}$ cells (C), $\mathrm{CD}^{+}{ }^{+} \mathrm{T}$ helper cells (D), neutrophil elastase (E), and negative (F). Arrows highlight examples of positively stained cells; arrowheads highlight positively stained cells in the intervillous space. Scale bar $=50 \mu \mathrm{m}$. Original magnification, $\times 100$. VUE, villitis of unknown etiology.

Biobank and used as controls. All placentas were taken from mothers who showed no evidence of conditions such as preeclampsia or diabetes. Detailed case note review showed no clinical, biochemical, hematologic, or microbiological evidence of infection was present. Multiple pregnancies, abnormal fetal heartbeat (live birth only), and fetuses with anomalies were excluded.

\section{Sample Collection}

Placentas from cases of stillbirth had two to seven randomly selected samples per placenta obtained at the time of postmortem for histologic analysis. For live births three samples of placental villous tissue were taken, one each from the center, middle, and periphery of the tissue. ${ }^{19}$ Biopsies were fixed in neutral-buffered formalin for 24 hours before being processed and embedded in paraffin wax.

\section{IHC}

For immunohistochemistry (IHC), 5- $\mu \mathrm{m}$ serial sections were cut and mounted onto slides coated with poly-L-lysine (Sigma-Aldrich, Poole, UK). Immunostaining with colorimetric detection was performed as described previously. ${ }^{20}$ VUE lesions were initially identified by detecting clusters of leukocytes with the use of a mouse monoclonal antibody to CD45 (leukocyte common antigen). A VUE lesion was defined as an area of villous tissue with evidence of an extensive $\mathrm{CD}_{4} 5^{+}$cell infiltrate that displayed the morphologic properties of macrophages and lymphocytes. Subsequent immunostaining for specific immune cells was performed to identify $\mathrm{CD} 4^{+} \mathrm{T}$ cells, $\mathrm{CD} 8^{+} \mathrm{T}$ cells, $\mathrm{CD} 163^{+}$macrophages, and neutrophils. Further immunostaining for cytokines IL-2, IL-4, IL-10, and IL-12 was performed on representative sections from all samples. Finally, staining for IL-6 and transforming growth factor (TGF)- $\beta$ was performed on selected stillbirth samples with VUE $(n=6)$ and healthy pregnancies $(n=6)$. Heat-mediated antigen retrieval was performed for all antibodies except anti-neutrophil elastase and anti-IL-10 monoclonal antibodies. Primary antibodies were applied at the working concentrations detailed in Table 1. Each tissue section had a corresponding negative control on which mouse or rabbit IgG (Sigma-Aldrich) was applied at the same concentration as the primary antibody. Each staining run included a positive control tissue as described in Table 1.

\section{Immunofluorescence}

The phenotype of the macrophages and T cells in the placentas/ lesions was investigated by dual immunofluorescence with combinations of immune cell markers CD4 and CD163 and cytokines IL-2, IL-12, or IL-4. Sections were fixed and processed as described in the previous section. Autofluorescence was quenched by 1 -hour incubation in $0.25 \%$ ammonia (SigmaAldrich) in $70 \%$ ethanol. Primary antibodies were applied at the concentrations described in Table 1 at $4^{\circ} \mathrm{C}$ overnight. Secondary antibodies (goat anti-mouse Alexa 568 or goat antirabbit Alexa 488; Life Technologies, Paisley, UK) were applied at $5 \mu \mathrm{g} / \mathrm{mL}$ and incubated for 30 minutes at room temperature. DAPI-containing aqueous mounting medium was used to apply coverslips.

\section{Image Capture and Analysis}

Tissue sections were visualized with the $10 \times$ objective of an Olympus BX41 light microscope (Southend-on-Sea, UK). Images were captured with QIcam Fast 1394 camera (QImaging, Surrey, BC, Canada) and Image Pro Plus 6.0 (Media Cybernetics Inc., Rockville, MD). Quantitative, unbiased image analysis was completed with HistoQuest image analysis software version 3.5 (TissueGnostics, Vienna, Austria). The pixels of images viewed in this software are converted to grayscale (0 to 256 scale) and assigned an arbitrary number relating to staining intensity. The image analysis software differentiates hematoxylin ${ }^{+}$and diaminobenzidine (DAB) ${ }^{+}$cells or areas that are recorded as events (cells/nuclei) or area of staining. Results are presented as histograms or dot plot scattergrams to which staining area and/or intensity cutoffs can be applied to differentiate between cell populations. Analysis variables were optimized such that the number of $\mathrm{DAB}^{+}$events and hematoxylin ${ }^{+}$events were counted.

Two analyses were performed. First, for characterization of VUE lesions in stillbirth, inflammatory foci were identified in CD45-stained sections, then the remaining immunostains were 

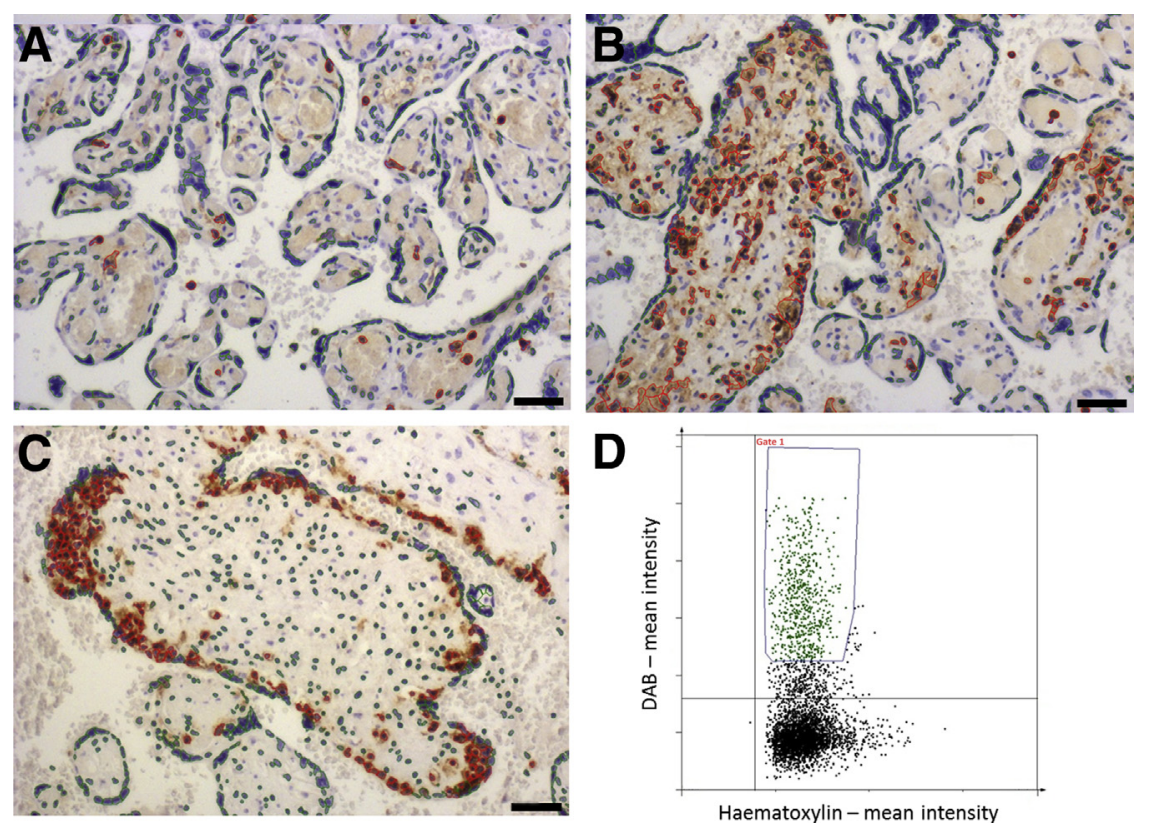

Figure 2 A-C: Screenshots from HistoQuest image analysis software demonstrating the selection of $\mathrm{DAB}^{+}$events in sections stained for CD45 leukocyte common antigen (A and $\mathbf{B}$ ) and cytotoxic T-lymphocyte marker CD8 (C). Positive events are highlighted in red; events encircled in green indicate hematoxylin positive/DAB ${ }^{-}$events. D: A representative scattergram from which staining intensity variables were ascertained. Scale bar $=$ $50 \mu \mathrm{m}$. Original magnification, $\times 100$. DAB, diaminobenzidine. matched and captured in serial sections. In tissue sections with widespread/numerous inflammatory foci, representative lesions were selected ( $n=2$ to 5 per section).

To assess pan-placental inflammation in tissue, 10 random images (excluding lesions) were taken for each placental slide from all groups (stillbirth with VUE, stillbirth with FGR, live birth with FGR, and controls), immunostained for CD45, CD163 macrophage, CD4 T cell, and CD8 T cell markers. $\mathrm{DAB}^{+}$cells and nuclei were quantified to ascertain whether there were any differences in leukocyte numbers between the study groups.

The presence of cytokines was determined by immunostaining for IL-2, IL-4, IL-6, IL-10, IL-12, and TGF- $\beta$. Images were analyzed quantitatively in sections of tissue from VUE lesions, areas of stillbirth with VUE placentas that were devoid of lesions, stillbirth with FGR placentas, live birth with FGR placentas, and controls $(n=10$ images per placenta). Area and intensity of staining were recorded for each placental sample, and quantitative analysis was completed with HistoQuest image analysis software.

Fluorescent-stained sections were visualized with a Zeiss fluorescence microscope, AxioCam MRn camera with Zen pro image processing software version 20.12 (Zeiss, Welwyn Garden City, UK).

\section{Statistical Analysis}

We powered our analysis to determine the number of $\mathrm{CD} 45^{+}$ and $\mathrm{CD}_{163}{ }^{+}$cells in placentas from stillborn infants, assuming similar increases in these cells as seen in placentas from poor outcome pregnancies experiencing reduced fetal movements. ${ }^{21}$ No similar studies have been conducted with CD4 and CD8 T cells, so we were not able to take direct comparisons. For $80 \%$ power with an $\alpha$ value of 0.05 , the sample size was calculated as 12. All statistical analysis was performed with GraphPad Prism software version 6.0 (GraphPad Software, San Diego, CA). Nonparametric categorical data were analyzed with Fisher's exact test; nonparametric continuous data were analyzed with $U$ test or Kruskal-Wallis test with Dunn's post hoc test.

\section{Results}

\section{Participant Demographic Characteristics}

The demographic data for the 48 women included in the study are shown in Table 2. A significant difference was found in maternal age between the stillbirth with FGR and normal pregnancy groups $(P=0.02)$. Contributory causes of death for the stillborn infant cohorts are detailed in Supplemental Table S1. By experimental design birth weight and individualized birth weight centile differences between stillbirth with FGR, live birth with FGR, and normal pregnancies were highly significant $(P<0.001$ and $P<0.01$, respectively). Average time between fetal death and delivery of infant was 3.5 days (range, 1 to 14 days). Comparisons of the grading/ extent of VUE between tissue samples could not be drawn because this information was not recorded in postmortem reports (Supplemental Table S2).

\section{Characterization of Focal Inflammatory Lesions}

VUE lesions were identified in all placentas from the stillbirth with VUE cohort; a representative lesion is shown in Figure 1. In total 104 VUE lesions from 12 placentas were photographed and analyzed. The number of $\mathrm{CD}^{4} 5^{+}$cells in the lesions contributed $25 \%$ (median; range, $8 \%$ to $48 \%$ ) to the total number of nuclei. Macrophages and $\mathrm{CD}^{+} \mathrm{T}$ lymphocytes were the most prevalent immune cells in the lesions, accounting for $12 \%$ (9\% to $23 \%$ ) and $11 \%$ (3\% to 


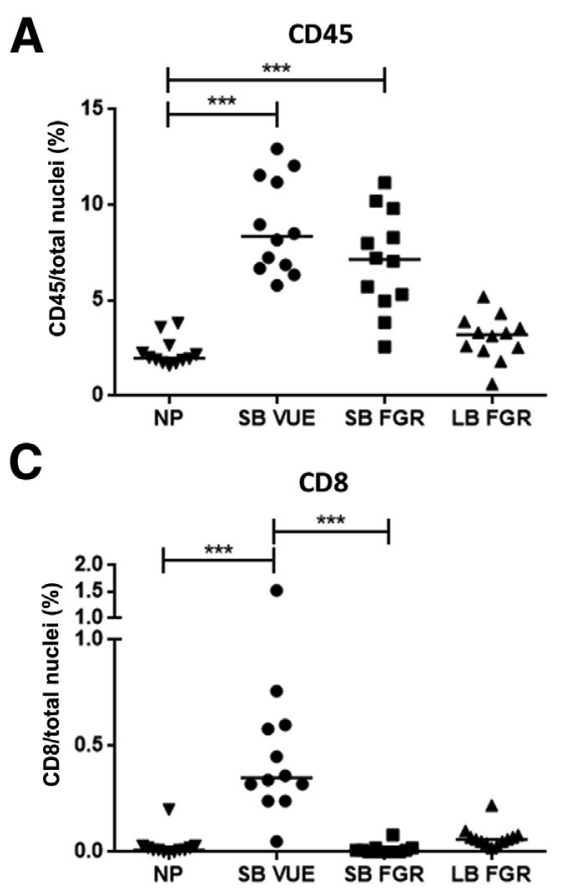

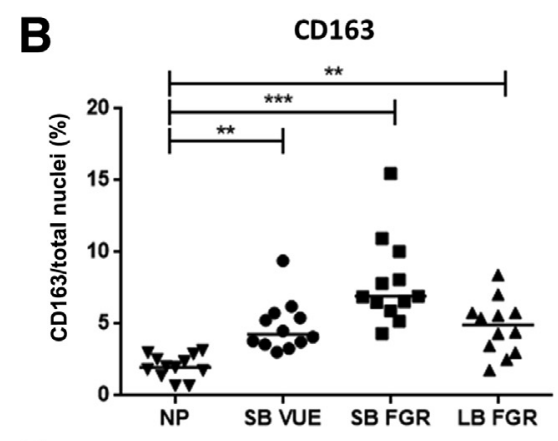

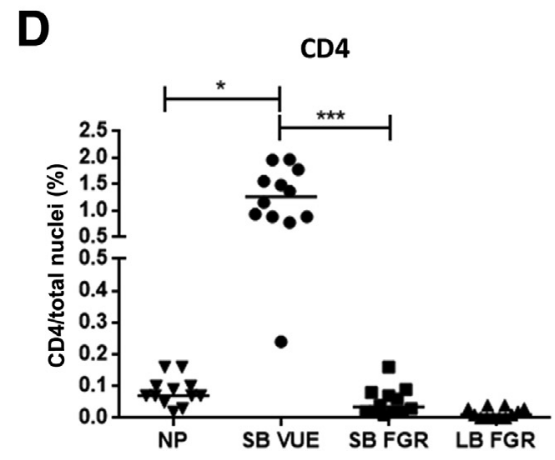

Figure 3 Quantification of pan-placental immune cells in normal and pathologic pregnancies. CD45 (A), macrophages (CD163, B), cytotoxic $T$ cells $(C D 8, C)$, and $T$ helper cells (CD4; D). Horizontal lines represent median. ${ }^{*} P<0.05$, ${ }^{* *} P<0.01$, and ${ }^{* * *} P<0.001$, Kruskal-Wallis test with Dunn's post hoc test. LB FGR, live birth with fetal growth restriction; NP, normal pregnancy; SB FGR, stillbirth with fetal growth restriction; $S B$ VUE, stillbirth with villitis of unknown etiology.
$14 \%$ ) of the total number of nuclei, respectively. $\mathrm{CD} 8^{+} \mathrm{T}$ lymphocytes were also present in VUE lesions, contributing $4 \%(0.3 \%$ to $11 \%)$ to the total number of nuclei. Neutrophil levels were low in all samples, accounting for $1 \%$ ( $0.37 \%$ to $2 \%$ ) of total number of nuclei.

\section{Differentiating Cell Populations}

Cell populations were differentiated by applying staining area and/or intensity cutoffs to the results presented as histograms or dot plot scattergrams (Figure 2).

\section{Quantification of Pan-Placental Immune Cells}

Significant increases were detected in the number of panplacental $\mathrm{CD} 45^{+}$leukocytes in all placentas from pregnancies from stillborn infants (with or without VUE) compared with normal pregnancies $(P<0.001)$ (Figure 3A). Significant elevation in macrophage numbers was detected in the placentas from stillborn infants with VUE $(P<0.01)$, stillborn infants with FGR $(P<0.001)$, and live born infants with FGR $(P<0.01)$ compared with normal pregnancy (Figure 3B). Placentas from the stillbirth with VUE group showed
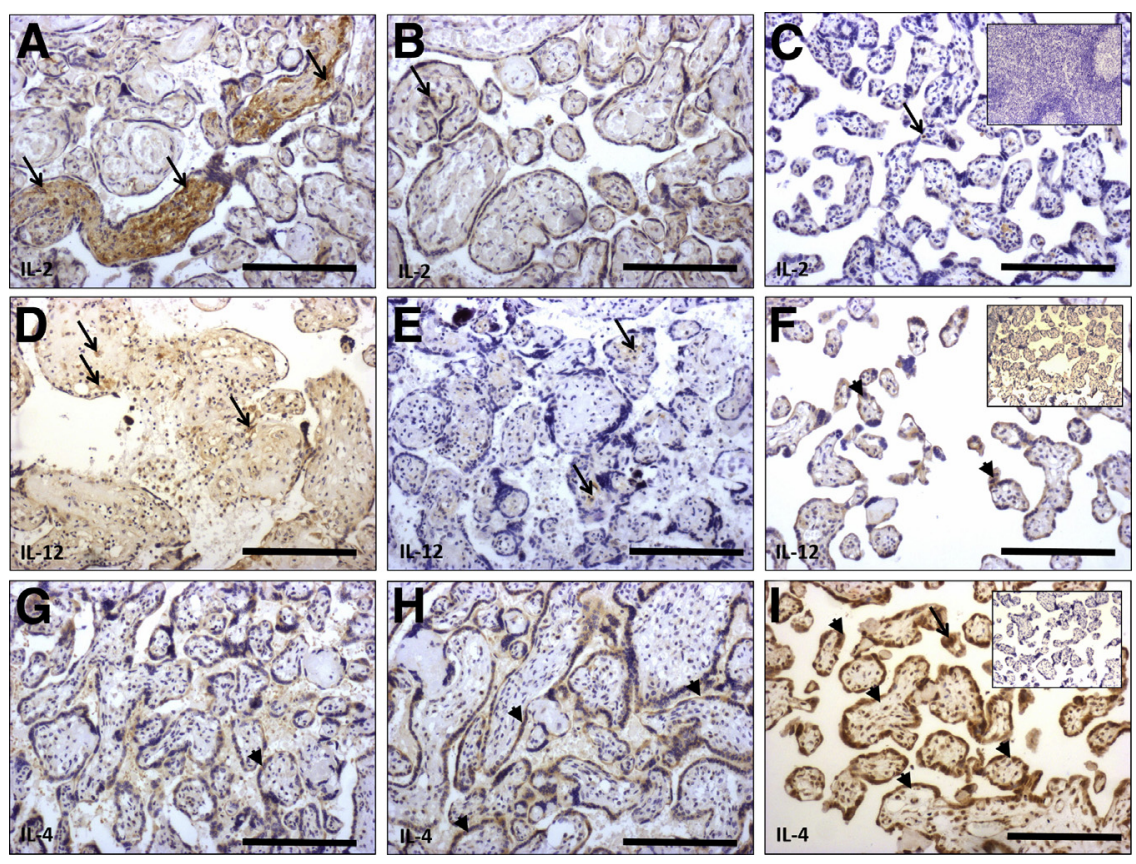

Figure 4 Representative images of placental sections immunostained for proinflammatory and anti-inflammatory cytokines. A, D, and G: VUE lesions were stained for IL-2 (A), IL-12 (D), and IL-4 (G). B, E, and H: Cytokine staining in areas of placenta unaffected by lesions from stillborn infants with VUE were stained for IL-2 (B), IL-12 (E), and IL-4 (H). C, F, and I: Representative sections of placenta from pregnancies with a normal outcome were stained for IL-2 (C), IL-12 (F), and IL-4 (insets, negatives) (I). Insets show negative control slides using IgG isotypes. Arrows highlight staining associated with cells; arrowheads highlight staining associated with trophoblast. Scale bar $=50 \mu \mathrm{m}$. Original magnification, $\times 100($ A-I and insets). VUE, villitis of unknown etiology. 


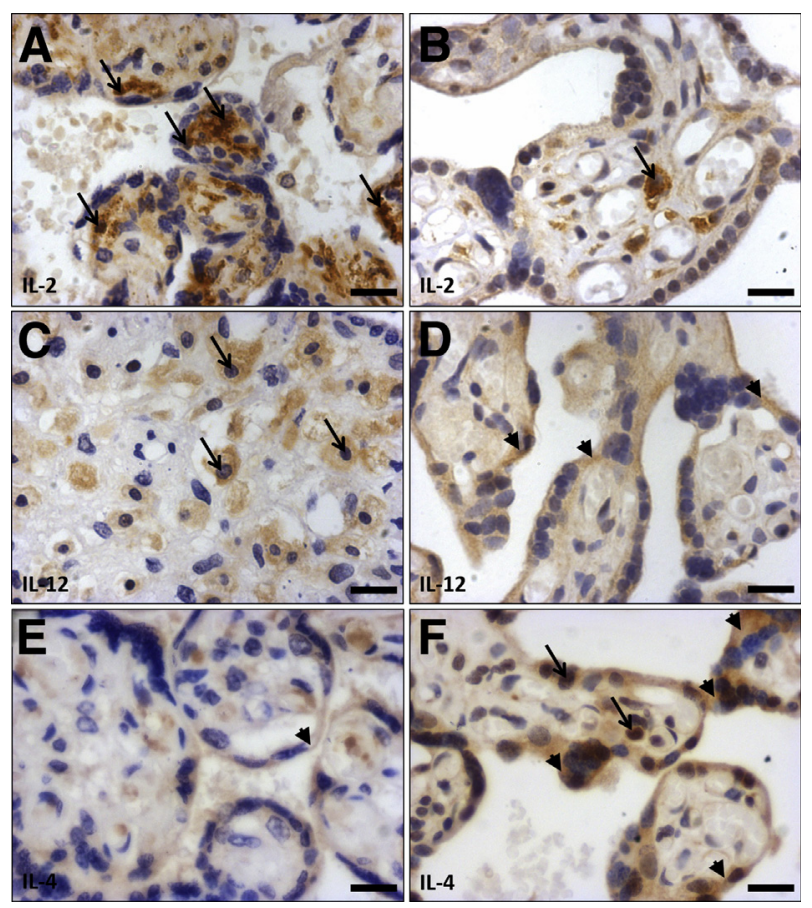

Figure 5 Representative high power images of placental sections after immunostaining for proinflammatory and anti-inflammatory cytokines. A, C, and E: VUE lesions were stained for IL-2 (A), IL-12 (C), and IL-4 (E). B, D, and F: Representative sections of placenta from pregnancies with a normal outcome were stained for IL-2 (B), IL-12 (D), and IL-4 (F). Arrows highlight staining of cells in the stromal core, arrowheads highlight staining associated with syncytiotrophoblast. Scale bar $=20 \mu \mathrm{m}$. Original magnification, $\times 400$. VUE, villitis of unknown etiology.

significantly more pan-placental CD4 and CD8 cells than all other groups $(P<0.001)$ and were observed in every field of view, excluding lesions (Figure 3, C and D).

\section{Cytokines}

To fully characterize the immune response in VUE, immunostaining of characteristic Th1 (IL-2, IL-12) and Th2 (IL-4) cytokines was performed (Figure 4). Staining of IL-2 and IL-12 localized to cells within the stromal core and stillborn, as previously shown ${ }^{22}$ (Figure 5). Area of IL-12 staining was significantly increased in VUE lesions and live born FGR placentas compared with normal pregnancy $(P<0.001)$. A significant increase in area of IL-2 staining was observed in VUE lesions $(P<0.05)$ (Figure 6). IL-4 staining localized to both stromal and trophoblast cells ${ }^{23}$ (Figure 5). A significant reduction was found in the area of IL-4 immunostaining in VUE lesions and areas devoid of lesions compared with controls $(P<0.05$ and $P<0.01$, respectively). A significant reduction in IL-4 staining area was also observed in the stillbirth with FGR group compared with live birth with FGR and controls $(P<0.05$ and $P<0.001$, respectively). IL-10 appeared significantly increased overall in areas of VUE placentas devoid of lesions $(P<0.001)$, but individual comparisons failed to reach statistical significance (Figures 4 and 6, Supplemental Figure S1). Staining intensity for all cytokines was significantly different between samples (Supplemental Figure S2). Intensity of IL-2 was significantly reduced (stillbirth FGR, $P=0.006$; live birth FGR, $P=0.0004)$ and IL-12 increased (stillbirth FGR, $P=0.0002$; live birth FGR, $P<0.001)$ in FGR samples compared with control. IL-4 intensity was significantly reduced in VUE lesions compared with control $(P=0.02)$. IL-10 staining intensity was significantly increased in VUE placentas compared with stillbirth with FGR (VUE lesion, $P=0.03$; VUE nonlesion, $P=0.01$ ). Immunostaining for cytokines IL-6 and TGF- $\beta$ was not detected in VUE lesions (Supplemental Figure S1).

\section{Macrophage and T-Cell Phenotype}

Dual immunofluorescence was performed to identify immune cell sources of cytokines of interest. IL-2 co-localized with $\mathrm{CD}^{+}$staining, $\mathrm{CD} 163^{+}$on the cell surface co-localized with cytoplasmic IL-12 staining, and IL-4 co-localized with CD4 ${ }^{+}$ (Figure 7). Evidence of faint IL-4 fluorescence was found in the syncytiotrophoblast layer as observed in the images of chromogen staining (Figures 4 and 7).

\section{Discussion}

This study has fully characterized VUE in placentas from stillborn infants by quantifying the immune cells found in the lesions by using unbiased image analysis software, after IHC to detect specific immune cell types. We have also demonstrated specific pan-placental inflammatory changes in stillbirths (with and without VUE), indicating that a more widespread immune reaction was occurring in areas of placenta without VUE lesions. Furthermore, our cytokine analyses indicate that the immune response in the lesions is directed toward Th1-type response.

Previous studies of VUE in live birth have described lesions comprising mainly fetal macrophages ${ }^{24}$ and maternal $\mathrm{CD} 4^{+18,25}$ or $\mathrm{CD} 8^{+12,14} \mathrm{~T}$ lymphocytes. Our results support these descriptions: in cases of stillbirth, macrophages and $\mathrm{CD}^{+}{ }^{+} \mathrm{T}$ cells predominate, although elevated numbers of CD8 cells are also present. A key strength of the current study is the use of immunostaining to detect specific immune cell types. Pathologists examining hematoxylin and eosin-stained sections would not be able to identify individual leukocyte subsets, which are clearly visible after immunostaining for leukocyte, macrophage, CD4, and CD8 cell markers. VUE has been likened to graft rejection or graft-versus-host disease. ${ }^{11-13}$ The phenotype that we observed, with $\mathrm{CD}^{+}$lymphocytes outnumbering $\mathrm{CD} 8^{+}$ lymphocytes, agrees with the 1989 study of Labarrere et $\mathrm{al}^{25}$ and supports this hypothesis because the T-helper cells would assume responsibility for directing the immune response toward a Th1-type response. ${ }^{26,27}$ The presence of cytotoxic CD8 T cells in the lesions may contribute to both 


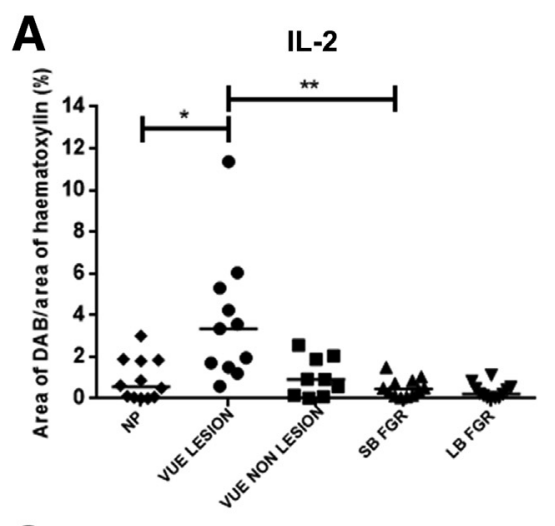

C

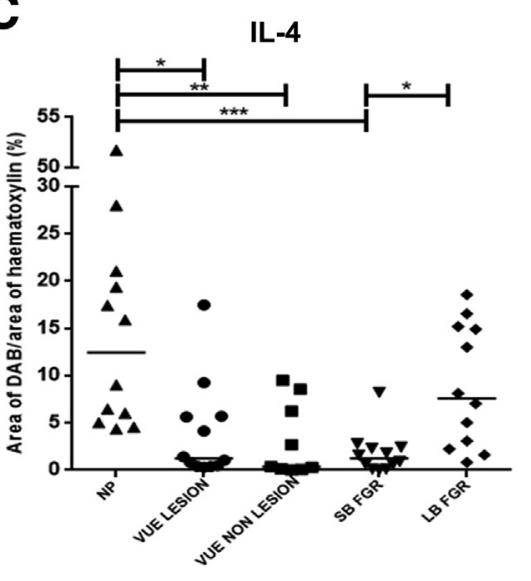

B

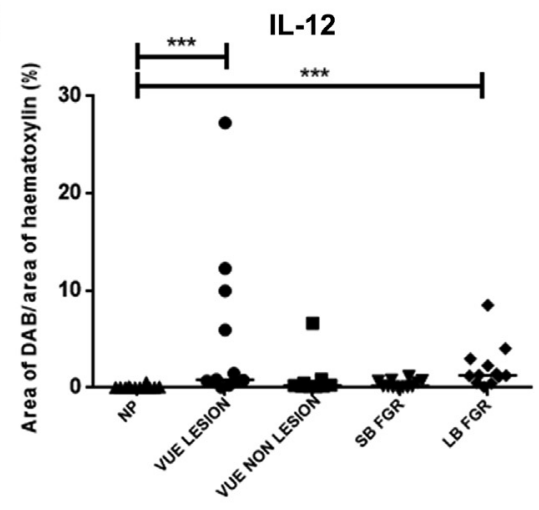

D

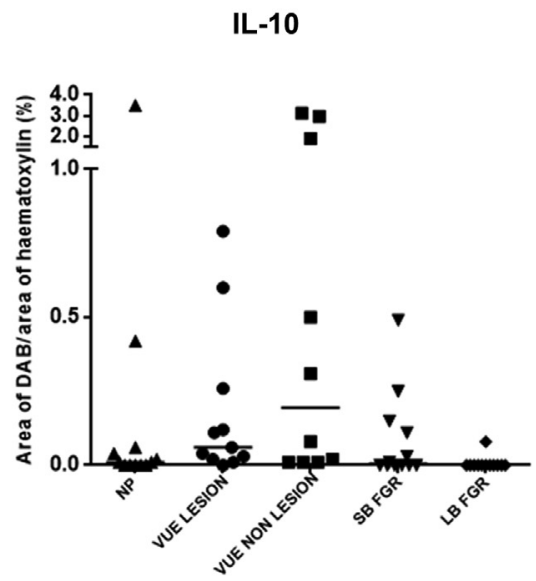

Figure 6 Quantification of cytokine staining area using HistoQuest image analysis software. A-D: IL-2 (A), IL-12 (B), IL-4 (C), and IL-10 (D). Horizontal lines represent median. ${ }^{*} P<0.05$, $* * P<0.01$, and ${ }^{* * * P} P<0.001$, Kruskal-Wallis test with Dunn's post hoc test. DAB, diaminobenzidine; LB FGR, live birth with fetal growth restriction; NP, normal pregnancy; SB FGR, stillbirth with fetal growth restriction; VUE, villitis of unknown etiology. the fetal placental vasculopathy often observed in VUE and the recently described elevated apoptosis of intravillous cells. $^{9,17,28,29}$

Since Medawar ${ }^{30}$ first described the immunologic paradox of pregnancy, much has been published on the shift from Th1 to Th2 responses in pregnancy. ${ }^{31-35}$ Suppression of anti-fetal cell-mediated responses and a skew toward antibody-mediated immunity appears to be advantageous in the maintenance of a successful pregnancy. ${ }^{35,36}$ The cytokine profile differs between Th1 and Th2 responses; IL-2, IL-6, and IL-12 are associated with Th1 responses, whereas anti-inflammatory cytokines IL-4, IL-10, and TGF- $\beta$ predominate in Th2-type responses. Our findings of increased levels of IL-2 and IL-12 in VUE lesions suggest that there may be an inappropriate shift toward cell-mediated immunity in VUE cases associated with stillbirth. Staining intensity of IL-2 and IL-12 was not significantly different between VUE samples and controls, implying that more cells are secreting the cytokine but the expression of it is not different. Co-localized immunofluorenscence staining of CD163/IL-12 and CD4/IL-2 is indicative of proinflammatory macrophage and Th1 CD4 T-cell phenotypes. We have also detected a significant reduction in levels of anti-inflammatory IL-4 in VUE placentas from cases of stillbirth. The reduction in IL-4 levels was mirrored by a significant reduction in expression in VUE lesions. The placental anti-inflammatory cytokine environment characterized by IL-4, IL-10, and TGF- $\beta$, which induces maternal tolerance of the fetus, is critical in maintaining a healthy pregnancy. ${ }^{32,36,37}$ Conversely, levels of proinflammatory $\mathrm{IL}-12$ remain low throughout successful pregnancy. ${ }^{36}$ Significant decreases in IL-4 in stillbirth placentas may be a contributory factor in permitting a shift from Th2-type to Th1-type immunity. These data support a reduction in Th2 and a Th1 bias in VUE lesions, which may lead to unresolved cell-mediated inflammation. Therefore, the critical balance appears to be disrupted in VUE lesions in cases ending in stillbirth.

It has been stated that macrophages and their cytokines are fundamental in establishing and maintaining the placenta at the start of pregnancy. ${ }^{38,39}$ It is therefore reasonable to suggest that an excess of immune cells and proinflammatory cytokines in late pregnancy may have a detrimental effect on placental function, which is then manifested as FGR or stillbirth. These effects would be in addition to the cytotoxic effects of any immune response mounted. Interestingly, systematic reviews suggest a general shift toward a proinflammatory cytokine profile in maternal serum and placentas from FGR and small for gestational age infants, confirming a role for a proinflammatory state in FGR. ${ }^{40,41}$ Conflicting reports of M2 placental macrophages potentially acting as either proinflammatory ${ }^{42}$ or immunosuppressive ${ }^{29}$ cells only serve to compound the mystery underlying VUE. Our investigation has shown placental macrophages expressing the M2 marker CD163, yet producing the proinflammatory cytokine IL-12, further enhancing the evidence of the 

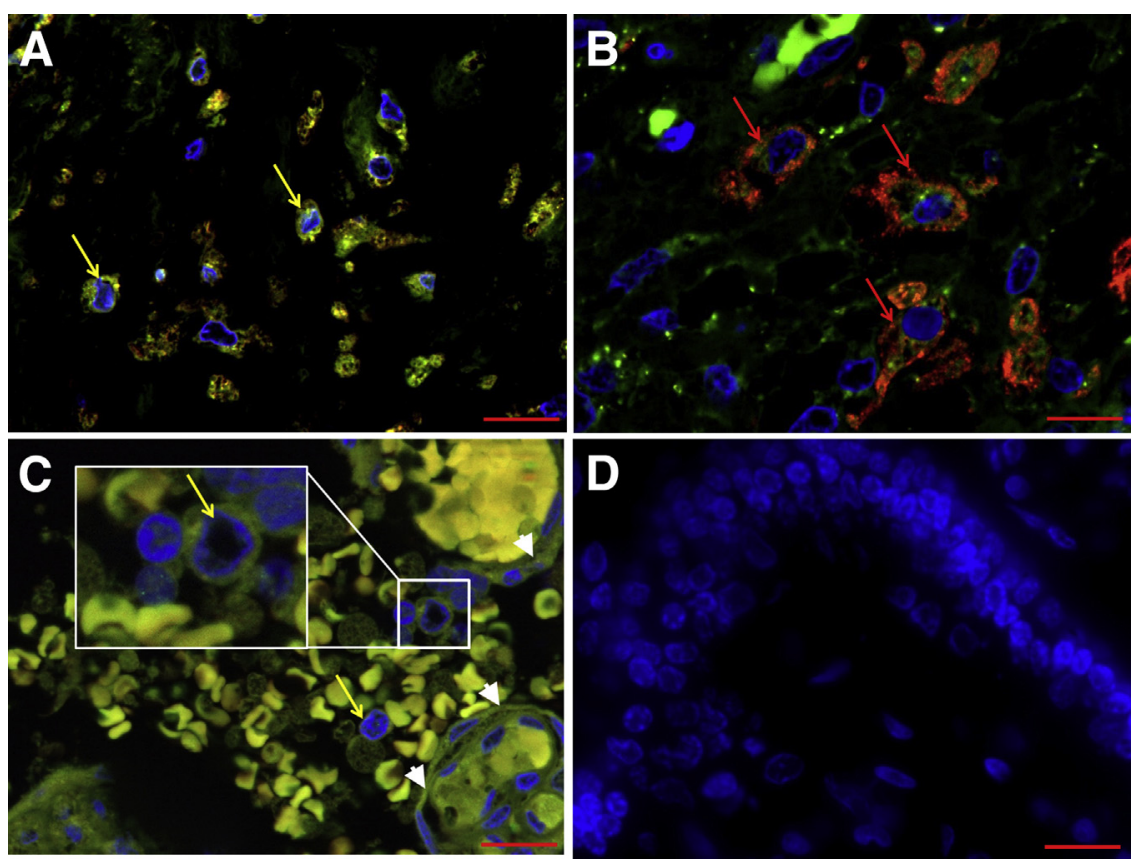

Figure 7 Representative high power images of placental sections after dual immunofluorescence for proinflammatory and anti-inflammatory cytokines and cell markers. A-C: VUE lesions stained for CD4 (red), IL-2 (green), co-localization (yellow) (A), CD163 (red) and IL-12 (green) (B), and CD4 (red) and IL-4 (green), co-localization (yellow) (C). D: Mouse and rabbit IgG isotype control. Red arrows highlight dual staining; yellow arrows indicate co-localized immunofluorescence; and white arrowheads indicate trophoblast-localized IL-4. Scale bar $=20 \mu \mathrm{m}$. Original magnification, $\times 630$ (A-D and inset). VUE, Villitis of unknown etiology.

plasticity of these cells and their potential role in pregnancy complications.

Pan-placental increases in leukocyte numbers in VUE are a novel finding. Significantly elevated numbers of leukocytes and macrophages were detected in all stillbirths compared with controls. The elevation in macrophages in live born FGR suggests that this is not a postmortem artifact. These data are consistent with our recent findings of elevated placental macrophage numbers in pregnancies at high risk of stillbirth. ${ }^{21}$ Staining intensity of cytokines in both live born and stillborn FGR were consistent throughout, further confirming that the structural characteristics observed is not related to postmortem tissue changes. The placentas from these pregnancies also exhibited a proinflammatory bias in relation to cytokine mRNA and protein expression (elevated IL-1 family members, reduced IL-10 and IL-4). Together, these data are highly suggestive of placental inflammation being an underlying factor in pregnancies that result in FGR and stillbirth.

Previous descriptions of VUE have stated the occurrence of focal villous lesions with adjacent unaffected tissue displaying normal structural characteristics. ${ }^{9}$ It has been suggested that the occurrence of VUE may have been underestimated because sampling of the placenta was not extensive enough to find evidence of lesions ${ }^{43}$; Altemani et $\mathrm{al}^{43}$ reported that at least six blocks are needed to achieve an $85 \%$ chance of diagnosing $95 \%$ of cases. In our study, CD4 and CD8 T-cell numbers were increased in stillbirths with VUE compared with all other group samples, and these increases were not confined to VUE lesions. This finding is a strength of the specificity of the IHC in our study. A sensitive and unbiased image analysis tool that can accurately detect immunostained cells with the use of a relatively low magnification $(\times 100)$ also contributes to the strength of the study. Our data suggest that a pan-placental increase in
CD4 and CD8 T cells should stimulate the pathologist to suspect an inflammatory condition, such as VUE, without relying on locating a specific lesion. With the use of IHC and sensitive image analysis software such as HistoQuest, the incidence of VUE could potentially be identified and accurately reported with fewer tissue samples. A general consensus on the sampling and definitions of placental lesions, as discussed by the Amsterdam Placental Workshop Group, attempts to address issues such as this (T.V. Khong et al, unpublished data). More sophisticated placental pathologic investigations may improve diagnosis of VUE (as was the case with estrogen receptor expression in breast cancer ${ }^{44}$ ) and thus provide much sought after explanations for the cause of death.

By examining placental tissue after delivery, IHC can only provide a snapshot of VUE, rather than a temporal profile of the cell migration and its associated effects. This could explain why descriptions of the proportions of T cells in VUE differ between research studies because they sample different periods in the evolution of lesions. ${ }^{12,14,17,18}$ In addition, the panplacental presence of CD4 and CD8 T cells may represent pre-lesions that, given time, would expand within the villous stroma, potentially explaining why VUE is considered a thirdtrimester phenomenon. ${ }^{9}$ The 1995 study by Khong, ${ }^{45}$ describing expression of major histocompatibility complex type II in both nonvillitis and villitis-affected placentas, also suggests that VUE may be an evolving lesion. This dynamic process cannot be surmised from the current method of studying placental tissue. The results of this study would be further enhanced by including samples of placenta from live born infants with evidence of VUE. At the time the study was conducted, these were not available.

A greater understanding of the immune responses associated with VUE may lead to the development of screening 
tests to enable antenatal detection of VUE. Our laboratory recently published data that indicate alterations in concentrations of maternal serum cytokines in pregnancies characterized by placental inflammation. ${ }^{21}$ This is supported by the observation of elevated maternal plasma chemokines (CXCL9, CXCL10, and CXCL11) in cases of VUE. ${ }^{13}$ It may therefore be possible to identify placental inflammation antenatally which would allow increased antenatal surveillance and potential anti-inflammatory therapies to prevent stillbirth.

\section{Conclusion}

We have fully characterized VUE in stillbirths with the use of IHC to identify specific immune cell types and have detected evidence of a Th1-type cytokine response. These data present novel insights into the pathogenesis of VUE and provide solid foundations for functional studies to determine the impact of inflammation on placental function.

\section{Acknowledgments}

We thank the mothers who gave consent to use the placentas that we studied, the recruiters, Dr. Mark Wareing who coordinated the Maternal and Fetal Health Research Group Biobank, Dr. Sylvie Girard for allowing us to use some of the immunostained sections from healthy pregnancies, Dr. Rachael Wright who dedicated a lot of time optimizing antibodies for IHC, and Dr. Joanne Pennock for immunologic advice.

This work forms part of a Ph.D. project for H.D.

\section{Supplemental Data}

Supplemental material for this article can be found at http://dx.doi.org/10.1016/j.ajpath.2015.12.010.

\section{References}

1. Flenady V, Middleton P, Smith GC, Duke W, Erwich JJ, Khong TY, Neilson J, Ezzati M, Koopmans L, Ellwood D, Fretts R, Frøen JF; Lancet's Stillbirths Series steering committee: Stillbirths: the way forward in high-income countries. Lancet 2011, 377:1703-1717

2. MacDorman M, Kirmeyer S: Fetal and perinatal mortality, United States, 2005. Natl Vital Stat Rep 2009, 57:1-19

3. Lawn JE, Blencowe H, Pattinson R, Cousens S, Kumar R, Ibiebele I, Gardosi J, Day LT, Stanton C; Lancet's Stilbirths Series steering committee: Stillbirths: Where? When? Why? How to make the data count? Lancet 2011, 377:1448-1463

4. Office of National Statistics: Statistical Bulletin: Births in England and Wales, 2013. Titchfield, United Kingdom, Office of National Statistics, 2014

5. Flenady V, Frøen JF, Pinar H, Torabi R, Saastad E, Guyon G, Russell L, Charles A, Harrison C, Chauke L, Pattinson R, Koshy R, Bahrin S, Gardener G, Day K, Petersson K, Gordon A, Gilshenan K: An evaluation of classification systems for stillbirth. BMC Pregnancy Childbirth 2009, 9:24
6. Heazell AEP, Worton SA, Higgins LE, Ingram E, Johnstone ED, Jones RL, Sibley CP: IFPA Gábor Than Award Lecture: recognition of placental failure is key to saving babies' lives. Placenta 2015, 36(Suppl 1): $\mathrm{S} 20-\mathrm{S} 28$

7. Ptacek I, Sebire NJ, Man JA, Brownbill P, Heazell AEP: Systematic review of placental pathology reported in association with stillbirth Placenta 2014, 35:552-562

8. Redline RW: Placental inflammation. Semin Neonatol 2004, 9: $265-274$

9. Redline RW: Villitis of unknown etiology: noninfectious chronic villitis in the placenta. Hum Pathol 2007, 38:1439-1446

10. Derricott H, Jones RL, Heazell AEP: Investigating the association of villitis of unknown etiology with stillbirth and fetal growth restriction a systematic review. Placenta 2013, 34:856-862

11. Rudzinski E, Gilroy M, Newbill C, Morgan T: Positive C4d immunostaining of placental villous syncytiotrophoblasts supports hostversus-graft rejection in villitis of unknown etiology. Pediatr Dev Pathol 2013, 16:7-13

12. Kim J-S, Romero R, Kim MR, Kim YM, Friel L, Espinoza J, Kim CJ: Involvement of Hofbauer cells and maternal $\mathrm{T}$ cells in villitis of unknown aetiology. Histopathology 2008, 52:457-464

13. Kim MJ, Romero R, Kim CJ, Tarca AL, Chhauy S, LaJeunesse C, Lee DC, Draghici S, Gotsch F, Kusanovic JP, Hassan SS, Kim J-S: Villitis of unknown etiology is associated with a distinct pattern of chemokine up-regulation in the feto-maternal and placental compartments: implications for conjoint maternal allograft rejection and maternal anti-fetal graftversus-host disease. J Immunol 2009, 182:3919-3927

14. Brito H, Juliano P, Altemani C, Altemani A: Is the immunohistochemical study of the inflammatory infiltrate helpful in distinguishing villitis of unknown etiology from non-specific infection villitis? Placenta 2005, 26:839-841

15. Redline RW, Patterson P: Villitis of unknown etiology is associated with major infiltration of fetal tissue by maternal inflammatory cells Am J Pathol 1993, 143:473-479

16. Kapur P, Rakheja D, Gomez AM, Sheffield J, Sanchez P, Rogers BB: Characterization of inflammation in syphilitic villitis and in villitis of unknown etiology. Pediatr Dev Pathol 2004, 7:453-458. discussion 421

17. Boog G: Chronic villitis of unknown etiology. Eur J Obstet Gynecol Reprod Biol 2008, 136:9-15

18. Katzman PJ, Murphy SP, Oble DA: Immunohistochemical analysis reveals an influx of regulatory T cells and focal trophoblastic STAT-1 phosphorylation in chronic villitis of unknown etiology. Pediatr Dev Pathol 2011, 14:284-293

19. Warrander LK, Batra G, Bernatavicius G, Greenwood SL, Dutton P, Jones RL, Sibley CP, Heazell AEP: Maternal perception of reduced fetal movements is associated with altered placental structure and function. PLoS One 2012, 7:e34851

20. Hamilton S, Oomomian Y, Stephen G, Shynlova O, Tower CL, Garrod A, Lye SJ, Jones RL: Macrophages infiltrate the human and rat decidua during term and preterm labor: evidence that decidual inflammation precedes labor. Biol Reprod 2012, 86:39

21. Girard S, Heazell AE, Derricott H, Allan SM, Sibley CP, Abrahams VM, Jones RL: Circulating cytokines and alarmins associated with placental inflammation in high-risk pregnancies. Am J Reprod Immunol 2014, 72:422-434

22. Bachmayer N, Rafik Hamad R, Liszka L, Bremme K, SverremarkEkström E: Aberrant uterine natural killer (NK)-cell expression and altered placental and serum levels of the NK-cell promoting cytokine interleukin-12 in pre-eclampsia. Am J Reprod Immunol 2006, 56: 292-301

23. de Moraes-Pinto MI, Vince GS, Flanagan BF, Hart CA, Johnson PM: Localization of IL-4 and IL-4 receptors in the human term placenta, decidua and amniochorionic membranes. Immunology 1997, 90: 87-94

24. Myerson D, Parkin RK, Benirschke K, Tschetter CN, Hyde SR: The pathogenesis of villitis of unknown etiology: analysis with a new 
conjoint immunohistochemistry-in situ hybridization procedure to identify specific maternal and fetal cells. Pediatr Dev Pathol 2006, 9: $257-265$

25. Labarrere C, Faulk W, McIntyre J: Villitis in normal term human placentae: frequency of the lesion determined by monoclonal antibody to HLA-DR antigen. J Reprod Immunol 1989, 16:127-135

26. Krieger NR, Yin DP, Fathman CG: CD4+ but not CD8+ cells are essential for allorejection. J Exp Med 1996, 184:2013-2018

27. Chaplin DD: Overview of the immune response. J Allergy Clin Immunol 2010, 125(2 Suppl 2):S3-S23

28. Feeley L, Mooney EE: Villitis of unknown aetiology: correlation of recurrence with clinical outcome. J Obstet Gynaecol 2010, 30: 476-479

29. Ito Y, Matsuoka K, Uesato T, Sago H, Okamoto A, Nakazawa A, Hata K: Increased expression of perforin, granzyme B, and C5b-9 in villitis of unknown etiology. Placenta 2015, 36:531-537

30. Billington WD: The immunological problem of pregnancy: 50 years with the hope of progress. A tribute to Peter Medawar. J Reprod Immunol 2003, 60:1-11

31. Challis JR, Lockwood CJ, Myatt L, Norman JE, Strauss JF 3rd, Petraglia F: Inflammation and pregnancy. Reprod Sci 2009, 16:206-215

32. Raghupathy R, Makhseed M, Azizieh F, Hassan N, Al-Azemi M, AlShamali E: Maternal Th1- and Th2-type reactivity to placental antigens in normal human pregnancy and unexplained recurrent spontaneous abortions. Cell Immunol 1999, 196:122-130

33. Cousins DJ, Lee TH, Staynov DZ: Cytokine coexpression during human Th1/Th2 cell differentiation: direct evidence for coordinated expression of Th2 cytokines. J Immunol 2002, 169:2498-2506

34. Perricone C, de Carolis C, Perricone R: Pregnancy and autoimmunity: a common problem. Best Pract Res Clin Rheumatol 2012, 26:47-60

35. Jamieson DJ, Theiler RN, Rasmussen SA: Emerging infections and pregnancy. Emerg Infect Dis 2006, 12:1638-1643

36. Lombardelli L, Aguerre-Girr M, Logiodice F, Kullolli O, Casart Y, Polgar B, Berrebi A, Romagnani S, Maggi E, Le Bouteiller P,
Piccinni M-P: HLA-G5 induces IL-4 secretion critical for successful pregnancy through differential expression of ILT2 receptor on decidual CD4 $^{+}$T cells and macrophages. J Immunol 2013, 191:3651-3662

37. Alijotas-Reig J, Llurba E, Gris JM: Potentiating maternal immune tolerance in pregnancy: a new challenging role for regulatory $\mathrm{T}$ cells. Placenta 2014, 35:241-248

38. Ingman K, Cookson VJ, Jones CJP, Aplin JD: Characterisation of Hofbauer cells in first and second trimester placenta: incidence, phenotype, survival in vitro and motility. Placenta 2010, 31: $535-544$

39. Burton GJ, Kaufmann P, Huppertz B: Anatomy and genesis of the placenta. Edited by Neill JD. The Physiology of Reproduction. ed 3. St. Louis, Elsevier, 2006, pp 211-266

40. Mullins E, Prior T, Roberts I, Kumar S: Changes in the maternal cytokine profile in pregnancies complicated by fetal growth restriction. Am J Reprod Immunol 2012, 68:1-7

41. Mullins E, Prior T, Roberts I, Kumar S: Changes in the fetal and neonatal cytokine profile in pregnancies complicated by fetal growth restriction. Am J Reprod Immunol 2013, 69:441-448

42. Young OM, Tang Z, Niven-Fairchild T, Tadesse S, Krikun G, Norwitz ER, Mor G, Abrahams VM, Guller S: Toll-like receptormediated responses by placental Hofbauer cells (HBCs): a potential pro-inflammatory role for fetal M2 macrophages. Am J Reprod Immunol 2015, 73:22-35

43. Altemani A, Gonzatti A, Metze K: How many paraffin blocks are necessary to detect villitis? Placenta 2003, 24:116-117

44. Barnes DM, Harris WH, Smith P, Millis RR, Rubens RD: Immunohistochemical determination of oestrogen receptor: comparison of different methods of assessment of staining and correlation with clinical outcome of breast cancer patients. Br J Cancer 1996, 74: $1445-1451$

45. Khong TY: Expression of MHC class II antigens by placental villi: no relationship with villitis of unknown origin. J Clin Pathol 1995, 48: 494-495 